\title{
Torus Knots Extremizing the Möbius Energy
}

\author{
Denise Kim and Rob Kusner
}

\section{CONTENTS}

1. Introduction

2. Möbius Invariance

3. Torus Knots and Symmetric Criticality

4. Torus Links

5. Discretization and Computer Experiments

6. Further Experiments

Acknowledgements

References

Software Availability
This work was accomplished while the authors were at the Institute for Advanced Study, and was partly supported by an NSF Postdoctoral Fellowship.

\begin{abstract}
Using the principle of symmetric criticality [Palais 1979], we construct torus knots and links that extremize the Möbiusinvariant energy introduced by O'Hara [1991] and Freedman, $\mathrm{He}$ and Wang [1993]. The critical energies are explicitly computable using the calculus of residues, a result obtained in collaboration with Gil Stengle.
\end{abstract}

Experiments with a discretized version of the Möbius energyapplicable to the study of arbitrary knots and links_-are also described, and confirm the results of the analytic calculations.

\section{INTRODUCTION}

Recently Freedman, He and Wang [Freedman et al.], following work of O'Hara [1991], introduced an energy $E(\Gamma)$ for a simple closed curve $\Gamma \subset \mathbf{R}^{3}$. The functional $E$ is continuous on each isotopy class of curves, and tends to infinity as $\Gamma$ nears self-intersection. Moreover, $E$ is "proper" on the set of all isotopy classes, in the sense that there are only finitely many knot types below a given energy level.

A useful geometric property of $E$ is Möbius invariance: if $\mu$ is a Möbius transformation of $\mathbf{R}^{3} \cup \infty$ and $\mu(\Gamma) \subset \mathbf{R}^{3}$, then $E(\mu(\Gamma))=E(\Gamma)$. This can be used to prove [Freedman et al.] that each prime knot class has an energy-minimizing representative (of differentiability class $C^{1,1}$ : see definition in the beginning of Section 2), and that the round circle is the unique energy minimizer among all curves, with $E=4$. It is also a nontrivial result of [Freedman et al.] that, at least for $C^{1,1}$ curves, the functional $E$ is sufficiently smooth to have a "gradient" $d E$ (see Section 3). Thus it becomes an interesting problem to find $E$-critical curves, that is, solutions to $d E=0$. 
In this article we construct the first explicit examples of knotted curves that are critical for $E$. Our basic observation is that the Möbius-invariant energy $E$ extends naturally to simple closed curves $\Gamma \subset \mathbf{R}^{m}$ (Section 2). In particular, if $\Gamma$ is a simple closed curve in $S^{3} \subset \mathbf{R}^{4}$ and $\sigma: S^{3} \rightarrow \mathbf{R}^{3} \cup \infty$ is stereographic projection, then $E(\sigma(\Gamma))=E(\Gamma)$ provided $\sigma(\Gamma) \subset \mathbf{R}^{3}$. Then we use the principle of symmetric criticality (Section 3 ) to show that for each relatively prime pair of integers $(p, q)$ there is a $(p, q)$-torus knot $\Gamma_{p, q} \subset S^{3}$ critical for $E$. This curve $\Gamma_{p, q}$ is a principal orbit of an isometric action of $S^{1}$ on $S^{3}$. In the same way, we construct critical orbital links with more than one component (Section 4).

The energies $E\left(\Gamma_{p, q}\right)$ can be computed explicitly in terms of rational trigonometric integrals. This makes them ideal benchmarks to check the accuracy of computer experiments with various discretized energies. One such discrete model is mentioned in Section 5. We have implemented several programs using this model, run experiments, and tabulated some of the results, but clearly more work needs to be done. In this regard, we mention that several Japanese mathematicians and, independently, Bryson [Bryson et al. 1993] have also conducted computer experiments to seek nontrivial extrema. In particular, Bryson reports finding a trefoil - that is, a $(2,3)$-torus knot-with energy approximately equal to 74 , which is close to the critical value of 74.4120 (see Table 1 in Section 5).

It is well-known (see [Schubert 1953, p. 250], for example) that torus knots are prime, and it is tempting to conjecture that the stereographic images $\sigma\left(\Gamma_{p, q}\right)$ are the energy minimizers guaranteed to exist by [Freedman et al.]. However, we expect this to be false when both $p$ and $q$ are large, for the following reason: We can view $\Gamma_{p, q}$ as a $p$ - or $q$-strand braid lying on the surface of a torus, and if both $p$ and $q$ are at least three, energy can be saved when "extra strands" of $\Gamma_{p, q}$ depart from the surface of the torus and push into the interiors of the complementary solid tori.
The right conjecture, which is supported by our most recent experiments (Section 6), appears to be this: $\Gamma_{p, q}$ is a stable local minimum for $E$ when $p=2$ or $q=2$, but is not a minimizer otherwise. The general $E$-stability question will be explored elsewhere [Kusner and Sullivan].

\section{MÖBIUS INVARIANCE}

Suppose $\Gamma \subset \mathbf{R}^{m}$ is a simple closed curve of class $C^{1,1}$, that is, one that admits a parametrization $\Gamma: S^{1} \hookrightarrow \mathbf{R}^{m}$ whose first derivative $\Gamma^{\prime}$ is Lipschitz. (Weaker differentiability assumptions are possible [Freedman et al., $\S 1$ ], but we find $C^{1,1}$ best suited to our purposes.) Let $D(x, y)$ denote the distance between $\Gamma(x)$ and $\Gamma(y)$ along $\Gamma$, that is,

$$
D(x, y)=\min \left(\int_{x}^{y}\left|\Gamma^{\prime}(z)\right| d z, \int_{y}^{x}\left|\Gamma^{\prime}(z)\right| d z\right) .
$$

Definition and Proposition. Given $\Gamma$ as above, the Möbius energy

$$
\begin{aligned}
E(\Gamma)=\iint_{S^{1} \times S^{1}}\left(\frac{1}{|\Gamma(x)-\Gamma(y)|^{2}}\right. & \left.-\frac{1}{D^{2}(x, y)}\right) \\
& \times\left|\Gamma^{\prime}(x)\right|\left|\Gamma^{\prime}(y)\right| d x d y
\end{aligned}
$$

is finite and independent of the parametrization of $\Gamma$. Moreover, if $\mu$ is a Möbius transformation of $\mathbf{R}^{m} \cup \infty$, then $E(\mu(\Gamma))=E(\Gamma)$ provided $\mu(\Gamma) \subset$ $\mathbf{R}^{m}$.

Proof. The arguments, with only formal modifications to move from 3 to $m$ dimensions, are essentially those of [Freedman et al., $\S \S 1$ and 2]. In particular, concerning the Möbius invariance, the first term in the integrand is pointwise invariant, whereas the second term in the integral is "intrinsic", in the sense that, for any $\varepsilon>0$, the asymptotic expansion

$$
\iint_{|x-y| \geq \varepsilon} \frac{\left|\Gamma^{\prime}(x)\right|\left|\Gamma^{\prime}(y)\right|}{D^{2}(x, y)} d x d y=\frac{4 \pi}{\varepsilon}-4+O(\varepsilon)
$$

is invariant under all $C^{1,1}$ diffeomorphisms of $\mathbf{R}^{m}$, not only Möbius transformations. 
Note that the $4 \pi / \varepsilon$ term here precisely cancels a corresponding singular term from the first integral. This is the "regularization" of $E$ introduced in [O'Hara 1991].

Stereographic projection extends to a Möbius transformation of $\mathbf{R}^{m+1} \cup \infty$, so we deduce:

Corollary. If $\Gamma \subset S^{m} \subset \mathbf{R}^{m+1}$ is a $C^{1,1}$ simple closed curve and $\sigma: S^{m} \rightarrow \mathbf{R}^{m} \cup \infty$ is stereographic projection, then $E(\sigma(\Gamma))=E(\Gamma)$ provided $\sigma(\Gamma) \subset \mathbf{R}^{m}$.

\section{TORUS KNOTS AND SYMMETRIC CRITICALITY}

For each pair $(p, q)$ of integers, there is an isometric action $\alpha_{p, q}$ of $S^{1}$ on the unit sphere $S^{3} \subset \mathbf{R}^{4}=\mathbf{C}^{2}$, defined by

$$
\alpha_{p, q}\left(e^{i \theta}\right):\left(\begin{array}{c}
z \\
w
\end{array}\right) \mapsto\left(\begin{array}{c}
e^{i p \theta} z \\
e^{i q \theta} w
\end{array}\right) .
$$

This action is effective when $p$ and $q$ are relatively prime, an assumption we make from now on.

The case $(p, q)=(1,1)$ is the familiar Hopf action, whose orbits are great circles in $S^{3}$. In general, the $\alpha_{p, q}$-orbits of the points $\left(\begin{array}{l}1 \\ 0\end{array}\right)$ or $\left(\begin{array}{l}0 \\ 1\end{array}\right)$ are $p$ - or $q$-fold coverings of the $z$ - or $w$-axis circles $S_{z}^{1}$ or $S_{w}^{1}$, respectively, while the remaining $\alpha_{p, q}$-orbits are embeddings $S^{1} \rightarrow S^{3}$. These principal orbits lie on tori

$$
T_{r}=\left\{(z, w):|z|=\frac{r}{\sqrt{1+r^{2}}},|w|=\frac{1}{\sqrt{1+r^{2}}}\right\},
$$

for $r>0$, where they have homology $(p, q)$ - that is, they are $(p, q)$-torus knots. Each torus is foliated by a circle's worth of congruent orbits; we can parametrize this family of orbits by associating to $e^{i t} \in S^{1}$ the orbit that goes through the point

$$
\left(\begin{array}{c}
e^{i m t} \frac{r}{\sqrt{1+r^{2}}} \\
e^{i n t} \frac{1}{\sqrt{1+r^{2}}}
\end{array}\right),
$$

where $m$ and $n$ are fixed integers such that

$$
\operatorname{det}\left(\begin{array}{cc}
m & n \\
p & q
\end{array}\right)= \pm 1
$$

We can think of $(r, t)$ as polar coordinates for the orbit space $\mathcal{S}_{p, q}$ of the action, which is an orbifold homeomorphic to $S^{2}$.

We denote by $\Gamma_{p, q}(r)$ an orbit of $\alpha_{p, q}$ lying in the torus $T_{r}$. For definiteness, we take the orbit corresponding to $t=0$, so that $\Gamma_{p, q}(r)$ is the knot

$$
e^{i \theta} \mapsto\left(\begin{array}{c}
e^{i p \theta} \frac{r}{\sqrt{1+r^{2}}} \\
e^{i q \theta} \frac{1}{\sqrt{1+r^{2}}}
\end{array}\right) .
$$

The Möbius energy of $\Gamma_{p, q}(r)$, which we denote by $E(r)$, is a smooth function of $r$ (see Figure 1). It turns out that we can compute $E(r)$ explicitly using residue calculus (see page 4 ); however, the results in this section do not depend on that computation.

Lemma. The knot $\Gamma_{p, q}(r)$ is critical for the Möbius energy $E$ if and only if

$$
\frac{\partial}{\partial r} E(r)=0
$$

Proof. The "only if" direction is clear. The "if" direction is a consequence of the principle of symmetric criticality, which states roughly that, for a functional that is invariant under some group $G$ of symmetries, the criticality of a $G$-invariant function need only be checked as if the functional were restricted to that part of its domain consisting of all $G$-invariant functions. See [Palais 1979], for example, for a general discussion.

More precisely, consider a $C^{1,1}$ map $V: S^{1} \rightarrow$ $\mathbf{R}^{4}$, thought of as a vector field along the knot $\Gamma=\Gamma_{p, q}(r)$, and a variation $\Gamma_{\varepsilon}=\Gamma+\varepsilon V$ of $\Gamma$. According to [Freedman et al., Lemma 6.1], there is a unique linear functional $d E$ such that

$$
d E(V)=\left.\frac{d}{d \varepsilon} E\left(\Gamma_{\varepsilon}\right)\right|_{\varepsilon=0},
$$

for any $V$ as above. (More formally, the Banach space of $C^{1,1}$ vector fields along $\Gamma$ is the tangent space to the Banach manifold of $C^{1,1}$ curves, and $d E$ is the gradient one-form of $E$ at $\Gamma$. Several explicit formulas for $d E$ are given in [Freedman et 


\title{
Computing the Möbius Energy of Torus Knots by Residue Calculus
}

\author{
Rob Kusner and Gil Stengle
}

To compute the Möbius energy $E(r)$ of the orbital torus knot $\Gamma_{p, q}(r)$ of $(3.2)$, we first rescale so the knot has the parametrization $\left(r e^{i p x}, e^{i q x}\right)$, and then use the definition of $E$ :

$$
\begin{aligned}
& E(r)=\int_{0}^{2 \pi} d y \int_{y-\pi}^{y+\pi} d x\left(\frac{r^{2} p^{2}+q^{2}}{r^{2}\left|e^{i p x}-e^{i p y}\right|^{2}+\left|e^{i q x}-e^{i q y}\right|^{2}}-\frac{1}{(x-y)^{2}}\right) \\
& =2 \pi \int_{-\pi}^{\pi} d s\left(\frac{r^{2} p^{2}+q^{2}}{r^{2}\left|e^{i p s}-1\right|^{2}+\left|e^{i q s}-1\right|^{2}}-\frac{1}{s^{2}}\right)
\end{aligned}
$$

where we have made the substitution $s=x-y$. In each of the cases $(p, q)=(1,0),(0,1)$ or $(1,1)$, the orbit $\Gamma_{p, q}(r)$ is a round circle, and we have, as already observed in [Freedman et al.],

$$
E\left(S^{1}\right)=2 \pi \int_{-\pi}^{\pi} d s\left(\frac{1}{\left|e^{i s}-1\right|^{2}}-\frac{1}{s^{2}}\right)=2 \pi \int_{0}^{\pi / 2} d \sigma\left(\frac{1}{\sin ^{2} \sigma}-\frac{1}{\sigma^{2}}\right)=2 \pi \frac{2}{\pi}=4,
$$

with $\sigma=\frac{1}{2} s$. Subtracting the first of these equalities from the second equality above for $E(r)$, we get

$$
E(r)-E\left(S^{1}\right)=2 \pi \int_{-\pi}^{\pi} d s\left(\frac{r^{2} p^{2}+q^{2}}{r^{2}\left|e^{i p s}-1\right|^{2}+\left|e^{i q s}-1\right|^{2}}-\frac{1}{\left|e^{i s}-1\right|^{2}}\right) .
$$

Using the identity $\left|e^{i x}-1\right|^{2}=-e^{-i x}\left(e^{i x}-1\right)^{2}$, valid for $x$ real, we see that the integrand can be extended to a meromorphic differential on $\mathbf{C}$ depending only on $e^{i s}$; the substitution $z=e^{i s}, d s=-i z^{-1} d z$ then expresses $E(r)$ as a contour integral along the unit circle, to which we can apply Cauchy's residue formula:

$$
\begin{aligned}
E(r) & =4+2 \pi \int_{-\pi}^{\pi} d s\left(\frac{-\left(r^{2} p^{2}+q^{2}\right)}{r^{2} e^{-i p s}\left(e^{i p s}-1\right)^{2}+e^{-i q s}\left(e^{i q s}-1\right)^{2}}-\frac{-1}{e^{-i s}\left(e^{i s}-1\right)^{2}}\right) \\
& =4+2 \pi \int_{|z|=1} d z\left(\frac{i\left(r^{2} p^{2}+q^{2}\right)}{r^{2} z^{1-p}\left(z^{p}-1\right)^{2}+z^{1-q}\left(z^{q}-1\right)^{2}}-\frac{i}{(z-1)^{2}}\right) \\
& =4+4 \pi^{2} \sum_{|z|<1} \operatorname{Res} \frac{-\left(r^{2} p^{2}+q^{2}\right)}{r^{2} z^{1-p}\left(z^{p}-1\right)^{2}+z^{1-q}\left(z^{q}-1\right)^{2}} .
\end{aligned}
$$

The last equality depends on the fact that the order-two singularity at $z=1$ of the first term in the integral is precisely cancelled by the second term. One can also check that the set of poles is invariant under conjugation and inversion in $S^{1}$. Moreover, the residues occur in conjugate pairs and have positive real part, so that each pole inside the unit disk contributes to $E$.

A similar calculation shows that the energy cross-term corresponding to two $(p, q)$-torus knots, with "polar coordinates" $(r, t)$ and $\left(r, t^{\prime}\right)$ in the notation of Section 3 , is

$$
4 \pi^{2} \operatorname{Res} \frac{-\left(r^{2} p^{2}+q^{2}\right)}{r^{2} z^{1-p} e^{-2 \pi i t}\left(z^{p}-e^{2 \pi i t}\right)^{2}+z^{1-q} e^{-2 \pi i t^{\prime}}\left(z^{q}-e^{2 \pi i t^{\prime}}\right)^{2}} .
$$

Thus, the total energy of the torus $\operatorname{link} \Gamma_{k p, k q}(r)$, for $p$ and $q$ relatively prime (see Section 4 for notation), is

$$
E(r)=k\left(4+4 \pi^{2} \sum_{j=0}^{k-1} \sum_{|z|<1} \operatorname{Res} \frac{-\left(r^{2} p^{2}+q^{2}\right)}{r^{2} z^{1-p} e^{-2 \pi i j m}\left(z^{p}-e^{2 \pi i j m}\right)^{2}+z^{1-q} e^{-2 \pi i j n}\left(z^{q}-e^{-2 \pi i j n}\right)^{2}}\right),
$$

where $m$ and $n$ are fixed integers such that $\operatorname{det}\left(\begin{array}{cc}m & n \\ p & q\end{array}\right)= \pm 1$ 
al., $\S 6]$, but its linearity is the only property we need here.)

Suppose that $\Gamma$ is not critical, so there exists a field $V$ such that $d E(V) \neq 0$. Since $\Gamma$ is an orbit of the $S^{1}$-action $\alpha=\alpha_{p, q}$, we can average the pushforward $\left(\alpha\left(e^{i \theta}\right)\right)_{*} V$ over $S^{1}$ to obtain an $\alpha$-invariant vector field $\tilde{V}$ for which $d E(\tilde{V}) \neq 0$ as well.

Now $\tilde{V}$ induces a variation through $\alpha$-orbits, so we can view $\tilde{V}$ as tangent to the orbit space $\mathcal{S}=$ $\mathcal{S}_{p, q}$ at $\Gamma$. But

$$
\left.d E\right|_{s}=\frac{\partial E}{\partial r} d r+\frac{\partial E}{\partial t} d t=\frac{\partial E}{\partial r} d r
$$

because knots with same $t$ have the same energy. Thus $\left.d E\right|_{\mathcal{S}}(\tilde{V})=d E(\tilde{V}) \neq 0$ implies $\partial E / \partial r \neq 0$, as required.

We come next to our main result.

Theorem 1. For each relatively prime pair of integers $p$ and $q$ greater than 1 , there is a $(p, q)$-torus knot $\Gamma_{p, q} \subset S^{3}$ that is critical for the Möbius energy $E$. The knot $\Gamma_{p, q}$ is a principal orbit of the $S^{1}$-action $\alpha_{p, q}$ defined above.

Proof. As already mentioned, $E(r)$ is a smooth function of $r$. Because $\Gamma_{p, q}(r)$ converges to either a $p$ - or $q$-fold covering of a round circle as $r$ approaches $\infty$ or 0 , and because $p, q \geq 2$, we have

$$
\lim _{r \downarrow 0} E(r)=\lim _{r \uparrow \infty} E(r)=+\infty .
$$

Thus there is a finite value $r_{p, q}>0$ minimizing $E(r)$. By the previous lemma, the principal orbit $\Gamma_{p, q}=\Gamma_{p, q}\left(r_{p, q}\right)$ is critical for $E$.

In fact, in all the examples we examined, there appears to be exactly one minimum (see Figure 1).

\section{TORUS LINKS}

In [Freedman et al.] the Möbius energy of a $k$ component link $\left(\Gamma_{1}, \ldots, \Gamma_{k}\right)$ is defined as a natural extension of the Möbius energy of a knot:

$$
E\left(\Gamma_{1}, \ldots, \Gamma_{k}\right)=\sum_{1 \leq i, j \leq k} E\left(\Gamma_{i}, \Gamma_{j}\right)
$$

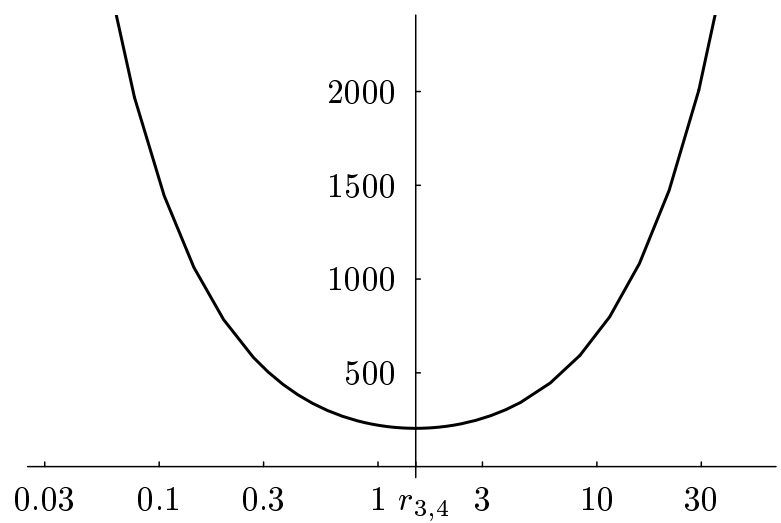

FIGURE 1. Graph of $E(r)$ versus $r$, for $(3,4)$-torus knots, from the formula on page 4 . Other values of $p$ and $q$ yield a very similar shape.

where $E\left(\Gamma_{i}, \Gamma_{i}\right)=E\left(\Gamma_{i}\right)$ is as before, and the crossterms $(i \neq j)$ are

$$
E\left(\Gamma_{i}, \Gamma_{j}\right)=\iint_{S^{1} \times S^{1}} \frac{\left|\Gamma_{i}^{\prime}(x)\right|\left|\Gamma_{j}^{\prime}(y)\right|}{\left|\Gamma_{i}(x)-\Gamma_{j}(y)\right|^{2}} d x d y .
$$

If we consider links composed of orbits of the $S^{1}$ action $\alpha_{p, q}$, the principle of symmetric criticality applies: a $k$-component orbital link is critical for $E$ among all links if and only if it corresponds to an $E$-critical configuration of $k$ distinct regular points in the orbit space $\mathcal{S}=\mathcal{S}_{p, q}$, that is, to a critical point of $E$ restricted to $\mathcal{S}^{k} \backslash \mathcal{D}$, where $\mathcal{D}$ is the big diagonal of $\mathcal{S}^{k}$ union the singular orbits. But $E$ is a positive proper function on $\mathcal{S}^{k} \backslash \mathcal{D}$, so it has a minimum.

In fact, it is easy to find some critical links explicitly. If $k>1$, denote by $\Gamma_{k p, k q}(r)$ the link consisting of $k$ copies of the knot $\Gamma_{p, q}(r)$, arranged symmetrically around the torus $T_{r}$. In other words, $\Gamma_{k p, k q}(r)$ is the union of the $\alpha_{p, q^{-}}$orbits with parameters $(r, t)$, with $t=0,2 \pi / k, \ldots, 2 \pi(k-1) / k$. (See the beginning of Section 3, and especially (3.1), for the $(r, t)$ parametrization of the orbit space.) We call $\Gamma_{k p, k q}(r)$ a $(k p, k q)$-torus link. Applying the principle of symmetric criticality with respect to the obvious action of $S^{1} \times C_{k}$, where $C_{k}$ is the cyclic group of order $k$, we conclude: 
Theorem 2. For each relatively prime pair of integers $p, q \geq 1$ and each $k>1$, there is a $(k p, k q)$ torus link $\Gamma_{k p, k q}$ which minimizes $E$ among $k$-component links invariant under $S^{1} \times C_{k}$, and which is therefore critical for $E$. In particular, there are infinitely many E-critical links with any number of components.

A $(k, k)$-torus link is a particular case of a Hopf link, that is, a link whose component curves are orbits of the Hopf action $\alpha_{1,1}$. A Hopf link can be regarded as a collection of points in the orbit space $\mathcal{S}_{1,1}=S^{2}$; the case of $\Gamma_{k, k}(r)$ corresponds to $k$ equidistant points along a parallel of latitude (circle of constant $r$ ). All $k$-component Hopf links are isotopic.

A direct calculation, or an application of $(*)$ on page 4 (with $r=1$ and $\alpha=t-t^{\prime}$ ), shows that the energy cross-term arising from an ordered pair of components of a Hopf link equals $2 \pi^{2} \csc \left(\frac{1}{2} \alpha\right)$, where $\alpha$ is the angular distance between the corresponding points in $S^{2}$. In particular, the total energy of a $k$-component Hopf link is, up to a constant, simply the "Coulomb energy" for $k$ point charges in $\mathbf{R}^{3}$ constrained to lie on $S^{2}$. (This observation was made jointly with John Sullivan.)

Since the points of $S^{2}$ corresponding to $\Gamma_{k, k}(r)$ are furthest apart when they are on the equator, that is, when $r=1$, Theorem 2 has the following consequence:

Corollary. For any $k$, the Hopf link $\Gamma_{k, k}(1)$ is $E$ critical.

In fact, $\Gamma_{2,2}(1)$, with energy $8+4 \pi^{2}$, is a minimizer among all nontrivial two-component links at least one component of which is a round circle; see [Freedman et al., $\S 7$ ]. One can also show that $\Gamma_{3,3}(1)$, with energy $12+8 \sqrt{3} \pi^{2}$, is energyminimizing among Hopf links with three components. In contrast, $\Gamma_{4,4}(1)$, with energy

$$
16+8(1+2 \sqrt{2}) \pi^{2} \approx 318.2805,
$$

is not minimizing among Hopf links with four components; the minimizing four-component Hopf link corresponds to a tetrahedral, rather than an equatorial, configuration of points on $S^{2}$, and its energy is $16+12 \sqrt{6} \pi^{2} \approx 306.1059$ (see Figure 2). This
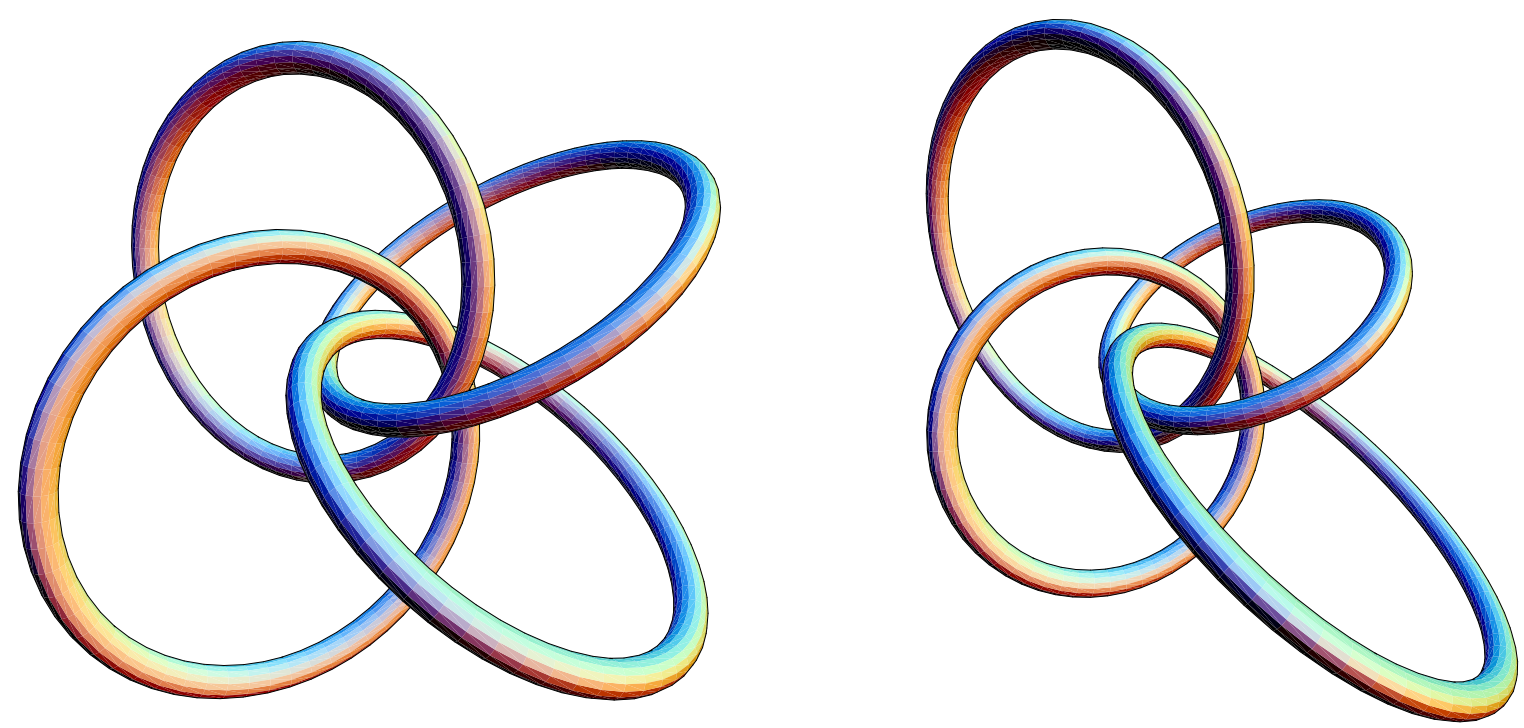

FIGURE 2. Two Hopf links critical for the energy functional $E$ : on the left, the torus link $\Gamma_{4,4}(1)$, whose components correspond (in the quotient space $S^{2}$ of the Hopf fibration) to four points equally spaced along a great circle; on the right, a link of less energy, whose components correspond to the vertices of a tetrahedron. See the corollary above and the subsequent paragraph. 
phenomenon illustrates the conjectured behavior, discussed in Section 1, of an $E$-minimizing $(p, q)$ torus knot for $p$ and $q$ large. Further results on Hopf links are given in [Kusner and Sullivan].

\section{DISCRETIZATION AND COMPUTER EXPERIMENTS}

Given a $C^{1,1} \operatorname{knot} \Gamma: S^{1}=\mathbf{R} / 2 \pi \mathbf{Z} \rightarrow \mathbf{R}^{m}$, and a sufficiently large integer $n$, the polygon $\gamma$ with vertices $\gamma(h)=\Gamma(2 \pi h / n)$, for $1 \leq h \leq n$, is a knot isotopic to $\Gamma$, which we call a polygonal approximation to $\Gamma$. One possible way to approximate $E(\Gamma)$ discretely would be simply to use $E(\gamma)$, but this energy unfortunately is infinite! It can be regularized, by deleting the contributions of adjacent edges, to an energy $E^{*}(\gamma)$ that has the advantage of an infinite barrier to changing isotopy type, but the disadvantage of being difficult to compute. Another discrete energy $e(\gamma)$, defined below, is much easier to compute. It can be shown that both $E^{*}(\gamma)$ and $e(\gamma)$ converge to $E(\Gamma)$ as $\gamma$ approaches $\Gamma$ for $n$ very large.

Definition. The discrete energy of a polygon with vertices $\gamma: \mathbf{Z} / n \mathbf{Z} \rightarrow \mathbf{R}^{m}$ is

$$
\begin{aligned}
e(\gamma)=\sum_{1 \leq i \neq j \leq n} \sum_{|\gamma(i)-\gamma(j)|^{2}}\left(\frac{1}{\mid d^{2}(i, j)}\right) & \\
& \times\left|\gamma^{\prime}(i)\right|\left|\gamma^{\prime}(j)\right|,
\end{aligned}
$$

where

$$
\left|\gamma^{\prime}(h)\right|=\frac{1}{2}(|\gamma(h-1)-\gamma(h)|+|\gamma(h)-\gamma(h+1)|)
$$

and $d(i, j)$ is the distance between $\gamma(i)$ and $\gamma(j)$ along the polygon, that is, the lesser of

$\sum_{i \leq h<j}|\gamma(h)-\gamma(h+1)|$ and $\sum_{j \leq h<i}|\gamma(h)-\gamma(h+1)|$.

If $\gamma=\gamma_{p, q}$ is a polygonal approximation to the orbit $\Gamma_{p, q} \subset S^{3}$, we can reduce the double sum above (by setting $h=i-j$ ) to

$$
e(\gamma)=n\left|\gamma^{\prime}(1)\right|^{2} \sum_{1<h \leq n}\left(\frac{1}{|\gamma(1)-\gamma(h)|^{2}}-\frac{1}{d^{2}(1, h)}\right) .
$$

Using this formula we wrote a simple Fortran search routine, torusknotenergy.f, to produce approximate values for the Möbius energies of critical $(p, q)$-torus knots. The results are very close to

\begin{tabular}{|c|c|c|c|c|}
\hline$(p, q)$ & $n$ & $\theta$ & $r_{p, q}$ & energy \\
\hline \multirow[t]{8}{*}{$(1,1)$} & 360 & 1.0 & - & 3.9608 \\
\hline & 720 & .5 & - & 3.9804 \\
\hline & 1440 & .25 & - & 3.9902 \\
\hline & 2880 & .12 & - & 3.9951 \\
\hline & 5760 & .06 & - & 3.9975 \\
\hline & 11520 & .03 & - & 3.9987 \\
\hline & extrapolati & ion & - & 3.9999 \\
\hline & analytic val & alue & - & 4.00000 \\
\hline \multirow[t]{6}{*}{$(2,3)$} & 1440 & .68 & 1.85 & 74.353 \\
\hline & 2880 & .34 & 1.85 & 74.383 \\
\hline & 5760 & .17 & 1.857 & 74.397 \\
\hline & 11520 & .09 & 1.857 & 74.405 \\
\hline & extrapolati & ion & 1.857 & 74.412 \\
\hline & analytic valu & ues & 1.857275 & 74.41204 \\
\hline \multirow[t]{6}{*}{$(2,8)$} & 1440 & .77 & 5.69 & 215.518 \\
\hline & 2880 & .38 & 5.69 & 215.646 \\
\hline & 5760 & .19 & 5.692 & 215.710 \\
\hline & 11520 & .10 & 5.693 & 215.742 \\
\hline & extrapolati & ion & 5.694 & 215.774 \\
\hline & analytic valu & ues & 5.694942 & 215.77463 \\
\hline \multirow[t]{6}{*}{$(3,4)$} & 1440 & .85 & 1.48 & 204.094 \\
\hline & 2880 & .42 & 1.48 & 204.156 \\
\hline & 5760 & .21 & 1.486 & 204.186 \\
\hline & 11520 & .11 & 1.486 & 204.201 \\
\hline & extrapolati & ion & 1.486 & 204.217 \\
\hline & analytic valu & ues & 1.486906 & 204.21670 \\
\hline
\end{tabular}
those obtained from the analytic formula on page 4 , which was implemented in a Mathematica program tke.m [Wolfram 1991]: see Table 1.

TABLE 1. Critical radii and energies obtained from the discrete programs torusknotenergy.f and toruslinkenergy.f. Here $n$ is the number of segments in the discretization, and $\theta$ is the maximum angle (in degrees) between segments. The last two lines in each block show how extrapolation of the program output as $n \rightarrow \infty$ gives results very close to those predicted by the analytic formula on page 4 , which were computed with the Mathematica programs tke.m and tle.m. 
These two programs are publicly available, together with their link counterparts, called tle.m and toruslinkenergy.f. For details, see "Software Availability" at the end of the article.

As a further check, we compared the computed values of $E$ and $r_{p, q}$ under an interchange of $p$ and $q$. As expected, $E$ remains the same, while $r_{p, q}$ is replaced by its reciprocal, to within the chosen accuracy. (In the case of tke.m, the default accuracy of the minimum search is six significant digits for $r$, but any desired accuracy can be specified, subject only to memory constraints.)

\section{FURTHER EXPERIMENTS}

Very recently, the second author and John Sullivan computed the gradient de of the discretized energy $e(\gamma)$, and Ken Brakke was kind enough to incorporate it into Version 1.89 of his Surface Evolver program [Brakke 1992]. Because $e$ and de are each quadratic in $n$, convergence of the negative gradient flow to a critical knot or link is considerably slower than in the program torusknotenergy.f, which searches only among orbits; the practical size of $n$ in the Evolver is limited accordingly. Nevertheless, we were able to test the stability of $\Gamma_{2, q}$ for $q$ small. Moreover, use of the Evolver allows us to search among all knots, not only those that are invariant under the $S^{1}$ action. We thus found a non-orbital $(3,4)$-torus knot with energy approximately 201 , less than the energy of $\Gamma_{3,4}$, which is 204.2167; see also the end of Section 1.

We hope to report further on this in future joint work with Brakke, Sullivan, and a group of students at the Five Colleges Geometry Institute.

\section{ACKNOWLEDGEMENTS}

We thank the School of Mathematics at the Institute for Advanced Study, the Center for Geometry, Analysis, Numerics and Graphics (G.A.N.G.) of the University of Massachusetts at Amherst, and the Geometry Center of the University of Minnesota, for the use of their computer facilities; and
Ken Brakke, Bruce Kleiner and John Sullivan, for their helpful comments. Thanks also to Charlie Gunn and Peter Norman for their interest in this project. We are grateful to Dick Palais and the referee for suggesting a number of improvements to the text, and to Mike Davis for pointing out the reference [Schubert 1953].

Finally, we would like to express our appreciation for Silvio Levy's superlative editing, which clarified the exposition, and for his contribution of the program tle.m, which completed the circle of ideas.

\section{REFERENCES}

[Brakke 1992] K. A. Brakke, "The Surface Evolver", Experimental Math. 1 (1992), 141-165.

[Bryson et al. 1993] S. Bryson, M. H. Freedman, Z. $\mathrm{X}$. He and Z. Wang, "Möbius Invariance of Knot Energy", Bull. Amer. Math. Soc. 28 (1993), 99-103.

[Freedman et al.] M. H. Freedman, Z. X. He and Z. Wang, "Möbius energy of knots and unknots", to appear in Annals of Math. Also available as "On the energy of knots and unknots", Research Report GCG 40, The Geometry Center, University of Minnesota.

[Kusner and Sullivan] R. Kusner and J. Sullivan, "A Möbius invariant, nonlocal energy for embedded submanifolds", in preparation.

[O'Hara 1991] J. O'Hara, "Energy of a knot", Topology 30 (1991), 241-247.

[Palais 1979] R. Palais, "The principle of symmetric criticality", Comm. Math. Physics 69 (1979), 19-30.

[Schubert 1953] H. Schubert, "Knoten und Vollringe", Acta Math. 90 (1953), 131-286.

[Wolfram 1991] S. Wolfram, Mathematica: A System for Doing Mathematics by Computer, 2nd ed., Addison-Wesley, Reading, MA, 1991.

\section{SOFTWARE AVAILABILITY}

The Mathematica programs tke.m and tle.m, as well as the Fortran programs torusknotenergy .f and toruslinkenergy.f, can be found in the directory pub/knot on the server polyphemus.gang. umass.edu. 
Denise Kim, Department of Physics, University of Massachusetts, Amherst, MA 01003 (kim@gang.umass.edu)

Rob Kusner, Department of Mathematics, University of Massachusetts, Amherst, MA 01003 (kusner@math.umass.edu)

Gil Stengle, Department of Mathematics, Lehigh University, Bethlehem, PA 18015 (gas0@lehigh.edu)

Received February 6, 1993; accepted in revised form April 27 